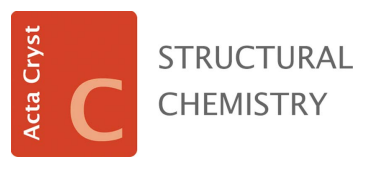

ISSN 2053-2296

\title{
A new tool for finding approximate symmetry
}

\author{
Carolyn Pratt Brock*
}

Department of Chemistry, University of Kentucky, Lexington, KY 40506-0055, USA. *Correspondence e-mail: cpbrock@uky.edu

Keywords: approximate symmetry; phase relationships; structural families.
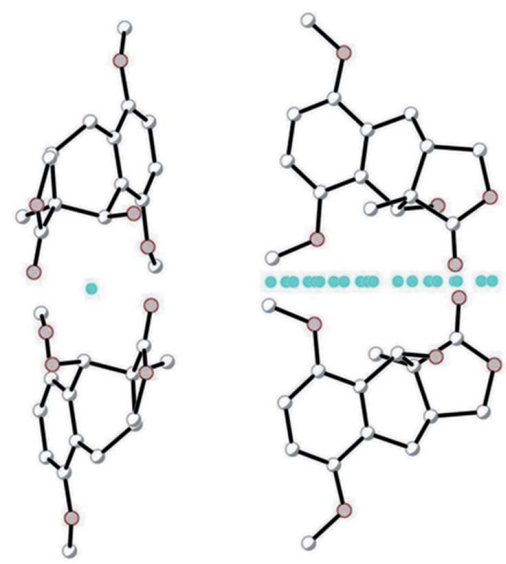

(C) 2019 International Union of Crystallography
Baggio's (2019) paper titled 'A simple graphical method to pinpoint local pseudosymmetries in $Z^{\prime}>1$ cases' should be studied by anyone interested in approximate symmetry, phase relationships, or structural families. Baggio's inventive approach is indeed simple, but it is also powerful, and it takes advantage of the sophisticated visualization software available [e.g. Mercury (Macrae et al., 2008) and XP (Sheldrick, 2008)].

All structures with $Z^{\prime}>1$ can be suspected of having some type of approximate (or occasionally even exact) symmetry in addition to the crystallographic symmetry reported. Approximate translations, screws, and inversions are common; approximate rotations and glides are known. Well over half the organic structures archived in the Cambridge Structural Database (CSD; Groom et al., 2016) with $Z^{\prime}>4$ and $R \leq 0.075$ have some kind of approximate symmetry (Brock, 2016). Of the $P 1, Z>1$ organic structures with $R \leq$ $0.050, \mathrm{ca} 10 \%$ would be better described in a higher-symmetry space group, while another $65 \%$ or so have approximate symmetry in three or two dimensions (Brock, 2019).

Approximate symmetry is often found in structures of crystals that have been cooled through a phase transition; an increase in the volume of the asymmetric unit during such transitions is common. Approximate symmetry, however, is also found in structures determined at room temperature. Possibly the crystal was grown at a higher temperature and then cooled through a transition, or maybe the crystal underwent a transformation during growth from a nucleus having higher symmetry.

Approximate symmetry that is not three dimensional is often termed local, but that term can be too limiting. Very good approximate symmetry in two-dimensional layers is not uncommon; in such cases, layer offsets lower the overall crystal symmetry. Consider the structure having the CSD refcode DUNHAW ( $P 1, Z=2$; Tang et al., 2010). Layers (011) in that structure have nearly exact $2_{1}$ axes along a. The other short axis in that layer, [011], makes an angle of $90.2^{\circ}$ with a; the approximate layer group is then $\not 2{ }_{1} 11$. Less often there is approximate symmetry that is confined to one dimension, as is the case for some hydrogen-bonded helices (e.g. the approximately $\not 5_{1} 12$ and $\not 5_{4} 12$ helices in refcode FEXCAN; Hao et al., 2005). Approximate symmetries of layers and rods can be described using the subperiodic groups listed in Vol. E of International Tables (Kopský \& Litvin, 2010).

For each pair of independent molecules Baggio's program calculates the mid-points of pairs of corresponding atoms. Those mid-points cluster around a point if the two molecules are related by approximate inversion; the better the approximate inversion the smaller the spread of the mid-points around the cluster center. Mid-points for two molecules related by an approximate twofold axis cluster around a line segment, with the cluster centroid lying on the line between the molecule centroids. If the molecules are related by a twofold rotation, the centroids of the molecules and of the mid-point cluster all lie in a plane perpendicular to the approximate axis; in the case of a twofold screw, the projections of the molecular centroids on the best line through the mid-point cluster are well separated along that line. Mid-point clusters for molecules related by an approximate glide or mirror are planar fragments, with glide and mirror relationships being distinguished by the location of that fragment relative to the two molecules. In the case of an approximate translation, the mid-points outline a 'ghost' molecule centered halfway between the two independent molecules.

Even very experienced crystallographers may have difficulties spotting and describing approximate symmetry elements, especially if they are not related to the crystal axes in a simple way. The cell constants may contain hints of higher symmetry, such as a monoclinic angle close to $90^{\circ}$, but the cell constants are not always good indicators. Baggio shows 
that LEBQIV $\left(P 2_{1}, \beta=103.8^{\circ}\right.$; Bume et al., 2017) has approximate symmetry $P 2{ }_{1} 2{ }_{1}{ }_{1}$, with $\mathbf{a}^{\prime}=\mathbf{a} / 2, \mathbf{b}^{\prime}=\mathbf{b}$, and $\mathbf{c}^{\prime}=\mathbf{a} /$ $2+$ c. We have found that SUQCUE $(P 1, Z=4 ; 11.4,12.4$, $16.0 \AA$ and $104.2,100.4,103.6^{\circ}$; Adero et al., 2015) has approximate symmetry $C 2, Z^{\prime}=1\left(\alpha=89.7, \gamma=90.3^{\circ}\right)$, with axes $\mathbf{a}^{\prime}=\mathbf{a} / 2+2 \mathbf{b}, \mathbf{b}^{\prime}=\mathbf{a} / 2$, and $\mathbf{c}^{\prime}=-(\mathbf{a} / 2+\mathbf{b}+\mathbf{c})$.

There is not yet any widely used software that detects approximate symmetry reliably. There does not seem to be any program at all that recognizes or characterizes approximate symmetry in layers or rods. While the oft-used ADDSYM routine in PLATON (Spek, 2009) is an excellent tool for finding unrecognized crystallographic symmetry, Baggio's article documents that $A D D S Y M$ is a less dependable tool for spotting approximate symmetry.

Baggio's program (MP, for Mid-Points) is available for download with the caveat that the program is 'not intended for distribution'. The paper is essentially a proof of concept, with detailed discussions of $20 Z^{\prime}=4$ structures. They include a member of a phase sequence, eleven structures having approximate symmetry, and eight structures suspected of having been refined in an inappropriate unit cell. Pictures of the mid-point clusters are given for all so that the spreads of the points can be seen.

The figures are very informative, but many readers will want to view and rotate sets of mid-points for themselves. The paper provides a link to .exe and .bat files, which were downloaded and run in this lab on a Windows computer without any problem. Sample input and output files are available with the supporting information. The .bat file includes information about program input, which includes several integers that control what is written to standard res files. As the article notes, it is helpful to suppress the clusters of mid-points corresponding to approximate translations because those points often obscure other features. The larger the spread of points in the cluster, the more difficult it is to recognize. Experience here with both the examples provided and with a number of other structures suggests that approximate twofold rotation and screw axes are the easiest to spot.

A drawback of the current version of the program is the requirement that the atom lists for the independent molecules be in exactly the same order. Incorporation of Baggio's approach into visualization programs that already include an atom-matching algorithm would solve that problem and would be an important step forward.

Since identification of approximate symmetry can be a judgement call, a quantitative measure based on analyses of the mid-point clusters will be important.

Extensions of the method to look for approximate symmetry in layers and rods seem straightforward; some tests in this lab have shown that the current version of $M P$ can be adapted easily to look at structures in which the approximate symmetry is confined to fewer than three dimensions.

Identifying approximate symmetry is an important step in finding structural relationships between phases of a compound. Sometimes the relationship is obvious, as is often the case when the crystal remains single through the transition. Sometimes, however, a reported new polymorph is a variant of a known structure. The structures in the refcode family LUXYOU provide an example of a relationship that might have been missed. At room temperature (RT), LUXYOU01 is disordered $\left(C 2 / c, Z=8, Z^{\prime}=1\right)$; at $100 \mathrm{~K}$ (LT), LUXYOU (Brandel et al., 2015) has a larger, lower-symmetry, ordered unit cell $\left(P 2_{1} / c, Z=48, Z^{\prime}=12\right)$. Layers (100) in the RT cell become layers $(10 \overline{1})$ in the LT form; the centering of the RT cell becomes an approximate translation [210]/4 in the larger cell. Some of the $2{ }_{1}$ axes of the RT phase are retained, while others become approximate, as do the twofold rotation axes. Identifying approximate symmetry can also be important for identifying structural families. Compounds that are very similar chemically may have different but related structures either because of the chemical difference or because data were collected at different temperatures. The family of simply substituted 4,4'-biphenyls (see Britton, 2005) is a good example.

Baggio's idea and program seem a very promising approach to a difficult problem.

\section{References}

Adero, P. O., Furukawa, T., Huang, M., Mukherjee, D., Retailleau, P., Bohé, L. \& Crich, D. (2015). J. Am. Chem. Soc. 137, 1033610345.

Baggio, R. (2019). Acta Cryst. C75, 837-850.

Brandel, C., Cartigny, Y., Couvrat, N., Eusebio, M. E. S., Canotilho, J., Petit, S. \& Coquerel, G. (2015). Chem. Mater. 27, 6360-6373.

Britton, D. (2005). Acta Cryst. C61, o35-o37.

Brock, C. P. (2016). Acta Cryst. B72, 807-821.

Brock, C. P. (2019). In preparation.

Bume, D. D., Pitts, C. R., Ghorbani, F., Harry, S. A., Capilato, J. N., Siegler, M. A. \& Lectka, T. (2017). Chem. Sci. 8, 6918-6923.

Groom, C. R., Bruno, I. J., Lightfoot, M. P. \& Ward, S. C. (2016). Acta Cryst. B72, 171-179.

Hao, X., Chen, J., Cammers, A., Parkin, S. \& Brock, C. P. (2005). Acta Cryst. B61, 218-226.

Kopský, V. \& Litvin, D. B. (2010). Editors, International Tables for Crystallography, Vol. E, 2nd ed., Subperiodic Groups. Chester: International Union of Crystallography.

Macrae, C. F., Bruno, I. J., Chisholm, J. A., Edgington, P. R., McCabe, P., Pidcock, E., Rodriguez-Monge, L., Taylor, R., van de Streek, J. \& Wood, P. A. (2008). J. Appl. Cryst. 41, 466-470.

Sheldrick, G. M. (2008). Acta Cryst. A64, 112-122.

Spek, A. L. (2009). Acta Cryst. D65, 148-155. 